\title{
What's left? An eye movement study of the influence of interword spaces to the left of fixation during reading
}

\author{
Timothy R. Jordan • Victoria A. McGowan • \\ Kevin B. Paterson
}

Published online: 12 February 2013

(C) Psychonomic Society, Inc. 2013

\begin{abstract}
In English and other alphabetic systems read from left to right, the useful information acquired during each fixational pause is generally reported to extend $14-15$ character spaces to the right of each fixation, but only 3-4 character spaces to the left, and certainly no farther than the beginning of the fixated word. However, this leftward extent is remarkably small and seems inconsistent with the general bilateral symmetry of vision. Accordingly, in the present study we investigated the influence of a fundamental component of text to the left of fixation-interword spaces - using a well-established eyetracking paradigm in which invisible boundaries were set up along individual sentence displays that were then read. Each boundary corresponded to the leftmost edge of a word in a sentence, so that as the eyes crossed a boundary, interword spaces in the text to the left of that word were obscured (by inserting a letter $x$ ). The proximity of the obscured text during each fixational pause was maintained at one, two, three, or four interword spaces from the left boundary of each fixated word. Normal fixations, regressions, and progressive saccades were disrupted when the obscured text was up to three interword spaces (an average of over 12 character spaces) away from the fixated word, while four interword spaces away produced no disruption. These findings suggest that influential information from text is acquired during each fixational pause from much farther leftward than is generally realized and that this information contributes to normal reading performance. Implications of these findings for reading are discussed.
\end{abstract}

Keywords Reading $\cdot$ Perceptual span · Interword spaces · Eye movements

T. R. Jordan $(\bowtie) \cdot$ V. A. McGowan • K. B. Paterson Faculty of Medicine, Biological Sciences and Psychology, University of Leicester, Leicester LE1 9HN, UK

e-mail: prof.timjordan@gmail.com
Normal reading relies on making saccadic eye movements along lines of text and ending each movement with a brief fixational pause, during which visual information is acquired. In this way, eye movements and fixational pauses provide visual information from a range of different areas on each line, and this contributes to the processing of text during reading (for a review, see Rayner, 2009).

However, the extent of the area at each fixational pause within which information influences reading (the perceptual span) appears to be quite limited. In particular, estimates of the perceptual span have been reported using an eyetracking technique in which a fixed area of text extending outward from each point of fixation is displayed normally during reading, but text outside this area is replaced (e.g., with $x \mathrm{~s}$ ). Using this technique, several studies have demonstrated that skilled readers of English, and of other alphabetic systems read from left to right, obtain useful information from an asymmetric area that extends $14-15$ character spaces to the right of fixation, but no more than 3-4 character spaces to the left, and certainly no farther than the beginning of the fixated word (e.g., McConkie \& Rayner, 1975, 1976; Rayner, Well, \& Pollatsek, 1980; Rayner, Well, Pollatsek, $\&$ Bertera, 1982). Consequently, while the perceptual span to the right of fixation is substantial, it is generally accepted that the perceptual span to the left of fixation is markedly small.

This asymmetry is viewed widely as demonstrating the involvement of forward-directed shifts of spatial attention during reading (e.g., Miellet, O’Donnell, \& Sereno, 2009; Pollatsek, Bolozky, Well, \& Rayner, 1981). Nevertheless, the view that normal reading uses information from such a short distance to the left of fixation seems rather odd. In particular, the human visual system shows clear bilateral symmetry in the acquisition of visual information to the right and left of fixation, and this symmetry extends across the visual field (for a review, see Jordan \& Paterson, 2009). 
Consequently, while the perceptual span extends rightward from fixation by some considerable distance, the same spatial extent of visual input that provides this rightward area of information during reading will also be available for text to the left of fixation. Moreover, it is clear that information supporting word recognition to the left of fixation can be acquired from areas substantially farther from fixation than the three to four character spaces indicated by the perceptual span. For example, Jordan, Patching, and Thomas (2003) presented words individually at various eccentricities to the right and left of a central fixation point and used an eyetracker to control the location of each presentation. The findings revealed that identification was better than chance for words to the right and left of fixation, even at an eccentricity of $4^{\circ}$, corresponding to about 14 character spaces from fixation. Since the processes involved in identifying words are a vital component of reading, useful visual information may well be acquired during reading from locations farther to the left of fixation than is generally accepted.

Some support for this view can also be found in studies of textual reading. Binder, Pollatsek, and Rayner (1999) found that changing the identity of the word to the left of the currently fixated word (and so, farther away than the widely reported leftward extent of the perceptual span) disrupted performance relative to when the word remained unchanged. In a similar vein, Rayner, Castelhano, and Yang (2009) found that performance was disrupted when the word to the left of the fixated word comprised only $x \mathrm{~s}$ during each fixational pause, and Apel, Henderson, and Ferreira (2012) found that regression performance was disrupted when the word to the left of the fixated word was composed of replacement letters. Thus, despite the widely cited parameters of the perceptual span, these findings suggest that reading can be influenced by information acquired farther to the left of fixation than is generally assumed.

Against this background, in the present research we took a fresh look at the size of the area to the left of fixation within which the properties of text influence reading performance. To do this, we chose a fundamental component of text - interword spaces - that several previous studies have shown exerts important influences to the right of fixation during reading. In particular, when interword spaces to the right of fixation were obscured in studies by replacing the spaces with other items (e.g., letters, digits, or blob-like gratings), reading was disrupted when the obscured text was within $12-15$ character spaces from fixation, suggesting that interword spaces from a substantial area of rightward text normally influence reading performance (e.g., McConkie \& Rayner, 1975, 1976; Morris, Rayner, \& Pollatsek, 1990; Pollatsek \& Rayner, 1982; see also Rayner, Fischer, \& Pollatsek, 1998). However, while influences of interword spaces to the right of fixation are well established, influences of interword spaces to the left of fixation have received very little attention. Indeed, only one study has specifically addressed the influence of interword spaces to the left of fixation (McConkie \& Rayner, 1976), but only three participants took part, and even the standard influences of rightward interword spaces were not obtained. Perhaps not surprisingly, this study also produced no discernible influences of leftward interword spaces on performance.

Accordingly, we investigated the influence of interword spaces to the left of fixation during reading using a version of the gaze-contingent boundary paradigm (e.g., Rayner, 1975 ) in which an invisible boundary was created for each word in a line of text (a normal sentence) presented on a screen. Each boundary was located at the screen coordinate corresponding exactly to the leftmost edge of a word in the sentence, so that as a saccade crossed a boundary during reading, selected interword spaces to the left of that boundary were obscured. In line with previous research using the boundary paradigm to investigate the perceptual span (for a review, see Schotter, Angele, \& Rayner, 2012), each change to interword spaces was made during a saccade (when the acquisition of information in reading is greatly suppressed) so that the implementation of each change was not visible to the reader.

Each interword space was obscured by replacing it with a letter $x$ (see Fig. 1). This procedure is highly effective at obscuring interword spaces in text whilst preserving all other information, and has been used extensively to establish the rightward extent of the influence of interword spaces on reading (Schotter et al., 2012). In the present study, all interword spaces to the left of fixation were obscured beginning at one of four locations (one, two, three, or four interword spaces) to the left of the fixated word. In the 1st-space condition, all leftward interword spaces were obscured from the first
Fig. 1 An illustration of the five types of display that were used. The $\mid$ symbol denotes the location fixated in this example (the symbol and the sentence are shown here for illustrative purposes and were not used in the experiment)

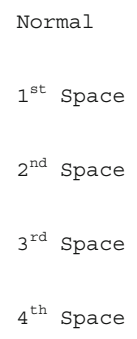

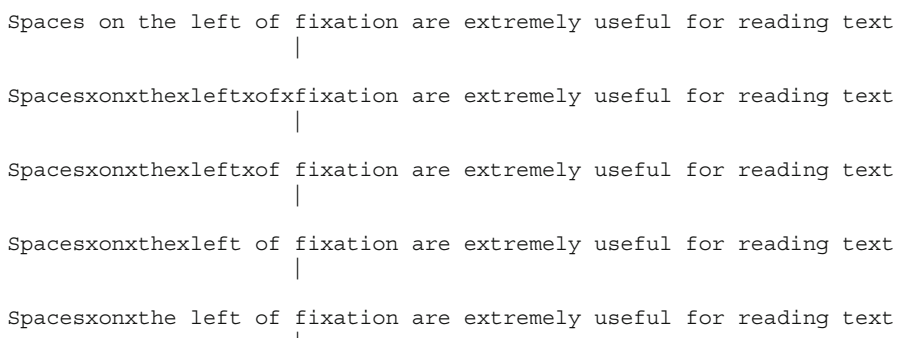


space to the left of the currently fixated word (between words $n$ and $n-1)$; in the $2 n d$-space condition, all leftward interword spaces were obscured from the second space to the left of the currently fixated word (between words $n-1$ and $n-2$ ); and so on. In this way, the proximity of the obscured interword spaces to each fixated word was controlled precisely so that the area to the left of fixation within which interword spaces influenced reading performance could be determined.

The logic of this research was straightforward. If processing interword spaces to the left of fixation is a normal component of reading, normal reading should be disrupted when these spaces are obscured. More crucially, the area within which these spaces influence reading should be revealed by the distance from fixation (one, two, three, or four interword spaces) at which obscuring interword spaces becomes disruptive. If the influence of information acquired to the left of fixation extends no farther than the left boundary of the currently fixated word (as the widely accepted view of the perceptual span proposes), reading performance should be disrupted, at best, only when information about this boundary is obscured (i.e., in the 1st-space condition). However, if the influence of interword spaces extends farther leftward than this, the area of this influence from fixation should become apparent. To provide a comprehensive measure of the influence of interword spaces on reading, reading performance was assessed by recording mean fixation duration, total number of fixations, progressive saccade count, regressive saccade count, progressive saccade length, regressive saccade length, and overall reading time.

\section{Method}

\section{Participants}

A group of 18 participants, 18-25 years of age, were recruited from the University of Leicester and the local community. All of the participants were native speakers of English and had normal or corrected-to-normal vision, as determined by the Bailey-Lovie (Bailey \& Lovie, 1980), ETDRS (Ferris \& Bailey, 1996), and Pelli-Robson (Pelli, Robson, \& Wilkins, 1988) assessments.

\section{Design and materials}

For the experiment we used a set of 144 sentences, and each sentence was presented as a single line of text. The sentences were 55-70 characters in length, of various structures, and did not include any syntactically anomalous items. The sentences contained an average of 11.3 words $(S D=1.3)$, and the average word length was 4.6 characters $(S D=2.4)$. Eighty sentences were selected pseudorandomly for each participant and displayed either as normal or in one of four display conditions in which a letter $x$ occupied all leftward interword spaces beginning at one of four locations from each fixated word: between the fixated word $n$ and word $n-$ 1 (1st space), between words $n-1$ and $n-2$ (2nd space), between words $n-2$ and $n-3$ (3rd space), and between words $n-3$ and $n-4$ (4th space). These locations appeared the following mean numbers of character spaces from the left boundary of the fixated word: 1 st space, 1.0; 2 nd space, 6.6 (SD 2.1); 3rd space, 12.2 (SD 3.1); and 4th space, 17.7 (SD 3.6). The sentence displays were randomized and selected for each display condition using a Latin square design, so that each participant saw any sentence only once, but all 144 sentences were shown equally often in each display condition across all participants. Practice sentences were presented at the start of each session.

\section{Apparatus and procedure}

The sentences were displayed as black text on a white background in Courier font on a 19-in. monitor with a screen resolution of $1,600 \times 1,200$ at $120 \mathrm{~Hz}$, and four letters subtended approximately $1.2^{\circ}$. Eye movements were recorded using an Eyelink $2 \mathrm{~K}$ eyetracker with a spatial resolution of $0.01^{\circ}$, and the position of each participant's right eye was sampled at $1000 \mathrm{~Hz}$ using corneal reflection and pupil tracking. An invisible boundary was located at a screen coordinate that corresponded exactly to the leftmost edge of each word in the sentence. As the eyes passed each invisible boundary, a letter $x$ was inserted in each appropriate interword space to the left of the invisible boundary before the next fixational pause. Since an invisible boundary was created for every word in a sentence, all letter $x$ s were displayed the appropriate number of interword spaces from the fixated word, whichever word was fixated. The time between crossing an invisible boundary and changing the sentence display was 6-8 ms. To check that the change to obscured interword spaces could not be detected when it was being implemented during a saccade, a pilot study was conducted in which the experimental displays were presented to five participants from the same population as those taking part in the experiment. These participants were asked to read each line of text and to report whether they could see any change in the display as it was made. All participants reported that the actual implementation of the changes during a saccade could not be detected.

At the beginning of the experiment, participants were instructed to read normally and for comprehension. The eyetracker was then calibrated. At the start of each trial, a fixation square (equal in size to one character) was presented at the left of the screen. Once the participant had fixated this location accurately for $250 \mathrm{~ms}$, a sentence was presented, with the first letter of the sentence replacing the 
square. Participants pressed a response key as soon as they had finished reading each sentence. The sentence was then replaced by a comprehension question, to which participants responded. Calibration was checked between trials, and the tracker was recalibrated as necessary.

\section{Results}

Participants showed high levels of sentence comprehension (>98\%), and a one-way analysis of variance comparing display conditions (normal and the four different space conditions) showed no differences in comprehension, $F(4$, $68)=0.53, p=.72, \eta_{\mathrm{p}}^{2}=.03$. Measures of reading performance were provided in terms of mean fixation durations (the average length of fixational pauses during reading), total numbers of fixations (the number of these fixational pauses), progressive saccade counts (the number of forward movements in the text), regressive saccade counts (the number of backward movements in the text), progressive saccade lengths, regressive saccade lengths, and overall reading times. The means for each of these measures are reported in Table 1 . A total of $6 \%$ of the data were removed due to the effects of blinks and track loss.

The purpose of this study was to determine whether performance with normal text would be influenced when interword spaces at various distances from fixated words (1st space, 2nd space, 3rd space, or 4th space) were obscured, and these influences were analyzed for each space condition using a one-way within-subjects analysis of variance for each measure of reading performance. Analyses were conducted first by using the whole-sentence data recorded from the start of each sentence. Mean fixation durations were longer than normal for the 1st-space, $F(1$, $17)=24.21, p<.0001, \eta_{\mathrm{p}}{ }^{2}=.59$, 2nd-space, $F(1,17)=$ 8.53, $p<.01, \eta_{\mathrm{p}}{ }^{2}=31$, and 3rd-space, $F(1,17)=13.19, p<$ $.01, \eta_{\mathrm{p}}{ }^{2}=.44$, conditions, and only the 4th-space condition showed no influence, $F<1.5, p>.20$. The number of fixations was also lower than normal for the 1st-space, $F(1,17)=12.21, p<.01, \eta_{\mathrm{p}}{ }^{2}=.42$, and 2nd-space, $F(1$, $17)=8.18, p=.01, \eta_{\mathrm{p}}{ }^{2}=.33$, conditions but not for the 3rdspace, $F=3.04, p=.10$, or 4th-space, $F<1$, conditions. Fewer progressive saccades than normal were also made in the 1st-space, $F(1,17)=5.71, p<.05, \eta_{\mathrm{p}}{ }^{2}=.21$, and 2 ndspace, $F(1,17)=8.45, p<.01, \eta_{\mathrm{p}}{ }^{2}=.30$, conditions but not for the 3 rd- and 4 th-space, $F_{\mathrm{S}}<1$, conditions. Fewer regressive saccades than normal were also made for the 1st-space, $F(1,17)=24.45, p<.0001, \eta_{\mathrm{p}}{ }^{2}=.59$, and 2nd-space, $F(1,17)=7.87, p=.01, \eta_{\mathrm{p}}{ }^{2}=.26$, conditions, but not for the 3 rd or 4 th space conditions, $F \mathrm{~s}<1.5, p \mathrm{~s}>.20$. Normal saccade lengths were unaffected by any of the space conditions, for both progressive (all $F_{\mathrm{s}}<2.2, p \mathrm{~s}>.15$ ) and regressive (all $\left.F_{\mathrm{S}}<3.3, p \mathrm{~s}>.09\right)$ saccades, and normal reading times were also unaffected by any space condition, all $F_{\mathrm{S}}<1.3, p \mathrm{~s}>.20$.

Analyses using the whole-sentence data naturally included data from the very beginning of sentences, before any interword spaces were obscured. Consequently, the influence of obscuring interword spaces was examined further using only data from the point in each display at which an $x$ appeared in an interword space. Thus, for the 1st-space condition, the analysis began from fixating the second word onward in each sentence, for the 2nd-space condition, from fixating the third word onward, and so on. The results supported the earlier analyses. Mean fixation durations were longer than normal for the 1st-space, $F(1,17)=21.72, p<$ $.001, \eta_{\mathrm{p}}{ }^{2}=.56,2$ nd-space, $F(1,17)=11.77, p<.01, \eta_{\mathrm{p}}{ }^{2}=$ .41 , and 3rd-space, $F(1,17)=14.15, p<.01, \eta_{\mathrm{p}}{ }^{2}=.45$, conditions, and only the 4th-space condition showed no influence, $F<2.16, p>.16$. The number of fixations was also lower than normal in the 1st-space, $F(1,17)=16.26$, $p<.001, \eta_{\mathrm{p}}{ }^{2}=.49$, and 2nd-space, $F(1,17)=13.01, p<.01$, $\eta_{\mathrm{p}}{ }^{2}=.43$, conditions but not in the 3 rd- and 4 th-space, $F_{\mathrm{S}}<$ $2.6, p>.12$, conditions. Fewer progressive saccades than normal were also made for the 1st-space, $F(1,17)=5.53$, $p<.05, \eta_{\mathrm{p}}{ }^{2}=.21$, and 2nd-space, $F(1,17)=10.63, p<.01$, $\eta_{\mathrm{p}}{ }^{2}=.39$, conditions but not for the 3 rd- or 4 th-space, $F \mathrm{~s}<1$,

Table 1 Eye movement measures for each interword space condition

\begin{tabular}{|c|c|c|c|c|c|}
\hline & \multicolumn{5}{|c|}{ Interword space condition } \\
\hline & Normal & 1st space & 2nd space & 3rd space & 4th space \\
\hline Fixation duration & 238 & 253 & 245 & 245 & 238 \\
\hline Number of fixations & 10.1 & 9.4 & 9.6 & 9.8 & 10.1 \\
\hline Number of progressive saccades & 7.5 & 7.2 & 7.2 & 7.4 & 7.4 \\
\hline Number of regressive saccades & 1.7 & 1.2 & 1.4 & 1.6 & 1.7 \\
\hline Progressive saccade length & 8.7 & 8.7 & 8.9 & 8.9 & 8.9 \\
\hline Regressive saccade length & 15.3 & 15.6 & 15.7 & 15.3 & 15.3 \\
\hline Reading time & 2,419 & 2,396 & 2,415 & 2,451 & 2,465 \\
\hline
\end{tabular}

Durations are given in milliseconds, and lengths are in characters 
conditions. Fewer regressive saccades than normal were also made in the 1 st-space, $F(1,17)=27.47, p<.0001, \eta_{\mathrm{p}}{ }^{2}=.62$, and 2 nd-space, $F(1,17)=7.86, p<.01, \eta_{\mathrm{p}}{ }^{2}=.32$, conditions, and marginally in the 3 rd-space condition, $F(1,17)=3.81, p=$ $.06, \eta_{\mathrm{p}}{ }^{2}=.18$, but not in the 4 th-space condition, $F<1$. Normal saccade lengths were unaffected by any of the space conditions for progressive saccades, all $F_{\mathrm{S}}<2.5, p \mathrm{~s}>.13$, and regressive saccades, all $F_{\mathrm{s}}<3.7, p \mathrm{~s}>.08$, and normal reading times were also unaffected by any space condition, all $F_{\mathrm{S}}<1$.

\section{Discussion}

The major indication from these findings was that obscuring interword spaces to the left of fixation during fixational pauses influenced reading performance even when the obscured spaces were no closer than two or three interword spaces (more than two words) from the fixated word. Thus, notwithstanding the general view that the perceptual span extends leftward no farther than the beginning of the fixated word, these findings indicate that influences on eye movement behavior during reading extend considerably farther to the left, and so occur more symmetrically around fixation. Indeed, three interword spaces in this study corresponded to an average of more than 12 character spaces, which is rather close to the well-established rightward extent of the influence of interword spaces during reading (e.g., McConkie \& Rayner, 1975, 1976; Morris et al., 1990; Pollatsek \& Rayner, 1982; see also Rayner et al., 1998). ${ }^{1}$

From previous studies of interword spaces, the influence of leftward interword spaces is likely to derive from two sources. Most clearly, interword spaces provide important information about the boundaries of words when reading (e.g., McConkie \& Rayner, 1975, 1976; Morris et al., 1990; Pollatsek \& Rayner, 1982; Rayner et al., 1998), and these boundaries affect the perception of global aspects of words, such as their spatial location and length. However, interword spaces can also facilitate word identification due to a reduction in lateral masking (e.g., Rayner et al., 1998), especially for the exterior letters of words, which may be particularly influential for reading (e.g., Jordan, 1995; Jordan, Thomas, Patching, \& Scott-Brown, 2003; Rayner, White, Johnson, \& Liversedge, 2006). Consequently, following the findings of the present research, information about the locations, lengths, and identities of words to the left of fixation may be acquired during each fixational pause.

\footnotetext{
${ }^{1}$ Although obscuring the 4th interword space from fixation did not influence normal performance, the influences observed for the 2nd and 3 rd interword spaces may have extended beyond these actual locations. This is because an obscured interword space will obscure the boundary of the word to the left of that location, and so perception of that farther word is likely also to be disrupted.
}

But although obscured interword spaces occurred only to the left of fixation in each display of this experiment, the pattern of effects observed on eye guidance indicates that this area of text exerts important influences on reading. In particular, as compared to normal text, obscuring leftward interword spaces produced longer fixations, fewer fixations, fewer regressive and progressive saccades, and no influence on the amplitude of either type of saccade. Consequently, the fundamental influence of obscuring leftward interword spaces appears to have been to suppress movement away from the fixated location, indicating that more time than normal was required for processing text during each fixational pause before the next saccade could be implemented.

This delay in moving the fixation location when leftward text was disrupted is consistent with previous views concerning the role of text passed during reading. In particular, it is often argued that information about words passed by a forward saccade, which are now to the left of the fixated word, may facilitate reading by providing important clues to the accuracy of the new fixation and its location within the same line (e.g., Reichle, Liversedge, Pollatsek, \& Rayner, 2009). In a similar vein, a number of researchers (e.g., Kennedy, Brooks, Flynn, \& Prophet, 2003) have proposed that when forward saccades are made, maintaining a record of words passed helps integrate information acquired at each new fixation with that acquired previously along the same line (see also Mitchell, Shen, Green, \& Hodgson, 2008). Consequently, when leftward information is disrupted (as in the present study), the information provided by this area of text during each fixation is likely to become more difficult to process, and so cause an increase in the duration of fixational pauses. Indeed, from the findings of the present study, information from text up to at least three interword spaces to the left of fixation may help maintain a cohesive mapping of the locations fixated during reading and help integrate new information acquired at each fixational pause with that already encountered along the same line.

But while obscuring leftward interword spaces clearly delayed shifts in fixation location, it did not increase the frequency with which text to the left of fixation was inspected. Indeed, as compared to normal text, obscuring leftward interword spaces produced fewer regressive and progressive eye movements and showed no influence on the amplitude of either type of saccade. Thus, while our findings suggest that information from words to the left of fixation is available when making regressive saccades (see also Baccino \& Pynte, 1994; Frazier \& Rayner, 1982; Kennedy et al., 2003), the influence of leftward text was not restricted to leftward eye movements. As a result, although it is well-established that obscuring interword spaces to the right of fixation disrupts normal reading performance (e.g., Morris et al., 1990; Pollatsek \& Rayner, 1982), normal forward progression also appears to be affected by similar 
manipulations to the left of fixation. Moreover, the finding that both regressive and progressive saccades were affected by text to the left of fixation makes it unlikely that these influences depend on leftward shifts of attention when reading. In contrast, the influences of text to the left of fixation that have been reported by other researchers may suggest that those influences occur only when a greater allocation of attention has been made to the left, and so occur with regressive saccades (Apel et al., 2012) and word skipping (Binder et al., 1999). Thus, while some aspects of eye movement behavior are associated with shifts in attention, the influence of leftward interword spaces that we observed in our study does not appear to rely on such shifts. ${ }^{2}$

We examined the extent to which the effects of obscuring leftward interword spaces relied on regressive saccades (Apel et al., 2012) and word skipping (Binder et al., 1999) by looking more closely at fixation durations. To do this, analyses of fixation durations were conducted as before, but now we excluded fixations made immediately before a regressive saccade and fixations made immediately after a word had been skipped. For whole-sentence data, mean fixation durations were longer than normal for the 1st-space, $F(1,17)=32.76$, $p<.0001, \eta_{\mathrm{p}}{ }^{2}=.66,2$ nd-space, $F(1,17)=5.52, p<.05, \eta_{\mathrm{p}}{ }^{2}=$ .24 , and 3rd-space, $F(1,17)=6.08, p<.05, \eta_{\mathrm{p}}{ }^{2}=.26$, conditions, and analyses using only data from the point at which interword spaces were obscured showed similar effects: 1st space, $F(1,17)=24.94, p<.0001, \eta_{\mathrm{p}}^{2}=.60 ; 2$ nd space, $F(1,17)=19.45, p<.0001, \eta_{\mathrm{p}}{ }^{2}=.53$; and 3rd space, $F(1,17)$ $=12.97, p<.01, \eta_{\mathrm{p}}{ }^{2}=.43$. Clearly, the indication is that the influences of leftward interword spaces on eye movement behavior do not rely on leftward shifts in attention.

Finally, it should be emphasized that although at first sight our findings may seem at odds with longstanding research concerning the leftward extent of the perceptual span, closer inspection (as we have pointed out) reveals surprisingly little previous evidence concerning the effect on reading exerted by interword spaces to the left of fixation. Consequently, while it may appear that previous research had provided a clear case against the importance of information to the left of fixated words when reading, our findings add to a growing body of evidence indicating that

\footnotetext{
$\overline{2}$ The findings of Apel et al. (2012) are particularly noteworthy, because they show no influences of leftward text on progressive saccades, and this represents a substantial difference from the findings of the present study. However, the data of Apel et al. were obtained using garden path sentences designed specifically to induce regressions, by containing a deliberate syntactic anomaly partway through that altered the meaning of the overall sentence. In contrast, the sentences used in the present study were not syntactically anomalous, and this may help explain the differences observed. Indeed, it may prove to be that when sentences in experiments are designed to induce regressions by using syntactic anomalies, strong leftward shifts in attention targeted at the required resolving locations suppress the more wide-ranging influences of leftward text that would otherwise be observed.
}

the leftward extent of the perceptual span is larger than is generally reported and widely assumed. However, our findings suggest that the influences of text to the left of fixation may be quite subtle (e.g., obscuring one, two, and three interword spaces from the fixated word increased normal fixation durations by an average of just $10 \mathrm{~ms}$ ), and so careful experimentation may be particularly necessary to ensure that these influences are not overlooked.

In sum, the findings of the present research show that key aspects of eye guidance during reading are affected by the availability of leftward interword spaces in an area extending up to three interword spaces (more than two words) away from the fixated word. Indeed, when this information is obscured during a fixational pause, normal progression through text is measurably affected. Accordingly, these findings indicate that rather than relying on an area to the left of fixation that is remarkably small when reading, the span of important information acquired during each fixational pause extends much farther to the left, and so is more symmetrical, than previously realized.

Author Note This research was supported by the Ulverscroft Foundation, a Professorial Research Fellowship from the Economic Research Foundation awarded to T.R.J., and a Mid-Career Research Fellowship from the British Academy awarded to K.B.P.

\section{References}

Apel, J. K., Henderson, J. M., \& Ferreira, F. (2012). Targeting regressions: Do readers pay attention to the left? Psychonomic Bulletin \& Review, 19, 1108-1113. doi:10.3758/s13423-012-0291-1

Baccino, T., \& Pynte, J. (1994). Spatial coding and discourse models during text reading. Language \& Cognitive Processes, 9, 143-155.

Bailey, I. L., \& Lovie, J. E. (1980). The design and use of a new nearvision chart. American Journal of Optometry and Physiological Optics, 57, 378-387.

Binder, K. S., Pollatsek, A., \& Rayner, K. (1999). Extraction of information to the left of the fixated word in reading. Journal of Experimental Psychology. Human Perception and Performance, 25, 1162-1172. doi:10.1037/0096-1523.25.4.1162

Ferris, F. L., \& Bailey, I. L. (1996). Standardizing the measurement of visual acuity for clinical research studies. Ophthalmology, 103, 181-182.

Frazier, L., \& Rayner, K. (1982). Making and correcting errors during sentence comprehension: Eye movements in the analysis of structurally ambiguous sentences. Cognitive Psychology, 14, 178-210. doi:10.1016/0010-0285(82)90008-1

Jordan, T. R. (1995). Perceiving exterior letters of words: Differential influences of letter-fragment and nonletter fragment masks. Journal of Experimental Psychology. Human Perception and Performance, 21, 512-530.

Jordan, T. R., Patching, G. R., \& Thomas, S. M. (2003). Assessing the role of hemispheric specialization, serial-position processing and retinal eccentricity in lateralized word perception. Cognitive Neuropsychology, 20, 49-71.

Jordan, T. R., \& Paterson, K. B. (2009). Re-evaluating split-fovea processing in word recognition: A critical assessment of recent 
research. Neuropsychologia, 47, 2341-2353. doi:10.1016/ j.neuropsychologia.2008.07.020

Jordan, T. R., Thomas, S. M., Patching, G. R., \& Scott-Brown, K. C. (2003). Assessing the importance of letter pairs in initial, exterior, and interior positions in reading. Journal of Experimental Psychology: Learning, Memory, and Cognition, 29, 883-893.

Kennedy, A., Brooks, R., Flynn, L.-A., \& Prophet, C. (2003). The reader's spatial code. In J. Hyönä, R. Radach, \& H. Deubel (Eds.), The mind's eye: Cognitive and applied aspects of eye movement research (pp. 193-212). Oxford, UK: Elsevier.

McConkie, G. W., \& Rayner, K. (1975). The span of the effective stimulus during a fixation in reading. Perception \& Psychophysics, 17, 578-586. doi:10.3758/BF03203972

McConkie, G. W., \& Rayner, K. (1976). Asymmetry of the perceptual span in reading. Bulletin of the Psychonomic Society, 8, 365-368.

Miellet, S., O'Donnell, P. J., \& Sereno, S. C. (2009). Parafoveal magnification: Visual acuity does not modulate the perceptual span in reading. Psychological Science, 20, 721-728.

Mitchell, D. C., Shen, X., Green, M. J., \& Hodgson, T. L. (2008). Accounting for regressive eye-movements in models of sentence processing: A reappraisal of the selective reanalysis hypothesis. Journal of Memory and Language, 59, 266-293.

Morris, R. K., Rayner, K., \& Pollatsek, A. (1990). Eye movement guidance in reading: The role of parafoveal letter and space information. Journal of Experimental Psychology. Human Perception and Performance, 16, 268-281.

Pelli, D. G., Robson, J. G., \& Wilkins, A. J. (1988). The design of a new letter chart for measuring contrast sensitivity. Clinical Vision Science, 2, 187-199.

Pollatsek, A., Bolozky, S., Well, A. D., \& Rayner, K. (1981). Asymmetries in the perceptual span for Israeli readers. Brain and Language, 14, 174-180.
Pollatsek, A., \& Rayner, K. (1982). Eye movement control in reading: The role of word boundaries. Journal of Experimental Psychology. Human Perception and Performance, 8, 817-833. doi:10.1037/ 0096-1523.8.6.817

Rayner, K. (1975). The perceptual span and peripheral cues in reading. Cognitive Psychology, 7, 65-81.

Rayner, K. (2009). The Thirty Fifth Sir Frederick Bartlett Lecture: Eye movements and attention during reading, scene perception, and visual search. Quarterly Journal of Experimental Psychology, 62, $1457-1506$.

Rayner, K., Castelhano, M. S., \& Yang, J. (2009). Eye movements and the perceptual span in older and younger readers. Psychology and Aging, 24, 755-760.

Rayner, K., Fischer, M. H., \& Pollatsek, A. (1998). Unspaced text interferes with both word identification and eye movement control. Vision Research, 38, 1129-1144. doi:10.1016/S0042-6989(97)00274-5

Rayner, K., Well, A. D., \& Pollatsek, A. (1980). Asymmetry of the effective visual field in reading. Perception \& Psychophysics, 27, 537-544. doi:10.3758/BF03198682

Rayner, K., Well, A. D., Pollatsek, A., \& Bertera, J. H. (1982). The availability of useful information to the right of fixation in reading. Perception \& Psychophysics, 31, 537-550.

Rayner, K., White, S. J., Johnson, R. L., \& Liversedge, S. P. (2006). Raeding wrods with jubmled lettres: There is a cost. Psychological Science, 17, 192-193. doi:10.1111/j.1467-9280.2006.01684.x

Reichle, E. D., Liversedge, S. P., Pollatsek, A., \& Rayner, K. (2009). Encoding multiple words simultaneously in reading is implausible. Trends in Cognitive Sciences, 13, 115-119. doi:10.1016/ j.tics.2008.12.002

Schotter, E. R., Angele, B., \& Rayner, K. (2012). Parafoveal processing in reading. Attention, Perception, \& Psychophysics, 74, 5-35. doi:10.3758/s13414-011-0219-2 